\title{
MEK Inhibition Induces Therapeutic Iodine Uptake in a Murine Model of Anaplastic Thyroid Cancer
}

\author{
Oussama ElMokh ${ }^{1}$, Vincent Taelman ${ }^{2}$, Piotr Radojewski ${ }^{2}$, Matthias A. Roelli ${ }^{1}$, Amandine Stoss ${ }^{1}$, Rebecca A. Dumont ${ }^{3}$, \\ Matthias S. Dettmer ${ }^{4}$, Wayne A. Phillips ${ }^{5}$, Martin A. Walter ${ }^{2}$, and Roch-Philippe Charles ${ }^{1}$ \\ ${ }^{1}$ Institute for Biochemistry and Molecular Medicine, University of Bern, Bern, Switzerland; ${ }^{2}$ Institute for Nuclear Medicine, Geneva \\ University Hospitals, Geneva, Switzerland; ${ }^{3}$ Department of Radiology, University of California at San Francisco, San Francisco, \\ California; ${ }^{4}$ Institute for Pathology, University of Bern, Bern, Switzerland; and ${ }^{5}$ Cancer Biology Laboratory, Peter MacCallum \\ Cancer Centre, Melbourne, Victoria, Australia
}

\begin{abstract}
Anaplastic thyroid carcinoma (ATC) is refractory to radioiodine therapy in part because of impaired iodine metabolism. We targeted the mitogen-activated protein kinase and phosphatidylinositol 3kinase $\left(\mathrm{PI} \mathrm{3}^{\prime} \mathrm{K}\right)$ pathways with the intent to induce radioiodine uptake for radioiodine treatment of ATC. Methods: Human ATC cells were used to evaluate the ability of pharmacologic inhibition of the mitogen-activated protein kinase and $\mathrm{PI}^{\prime} \mathrm{K}$ pathways to induce radioiodine uptake. Thyrocyte-specific double-mutant BRAFV600E PIK3CA ${ }^{\mathrm{H} 1047 \mathrm{R}}$ mice were treated with a MEK inhibitor followed by radioiodine treatment, and tumor burden was monitored by ultrasound imaging. Results: ATC cell lines showed an increase in sodium-iodine symporter transcription when treated with a MEK or BRAF ${ }^{\mathrm{V} 600 \mathrm{E}}$ inhibitor alone and in combination with $\mathrm{PI} \mathrm{3}^{\prime} \mathrm{K}$ inhibitor. This translated into a dose-dependent elevation of iodine uptake after treatment with a MEK inhibitor alone and in combination with a PI3'K inhibitor. In vivo, MEK inhibition but not BRAF or $\mathrm{PI} 3^{\prime} \mathrm{K}$ inhibition upregulated sodium-iodine symporter transcription. This translated into a stable reduction of tumor burden when mice were treated with a MEK inhibitor before radioiodine administration. Conclusion: This study confirms the ability of MEK inhibition to induce iodine uptake in in vitro and in vivo models of ATC. The approach of using a MEK inhibitor before radioiodine treatment could readily be translated into clinical practice and provide a much-needed therapeutic option for patients with ATC.
\end{abstract}

Key Words: drug resistance; NIS; targeted therapy; differentiation

J Nucl Med 2019; 60:917-923

DOI: 10.2967/jnumed.118.216721

\section{A}

naplastic thyroid carcinoma (ATC) is one of the most aggressive human malignancies $(1,2)$ and is refractory to radioiodine therapy (3).

Impaired iodine metabolism can result from dysregulation of the mitogen-activated protein kinase (MAPK) and phosphatidylinositol 3-kinase (PI3'K) pathways (Fig. 1), which regulate a variety of cellular activities and have been implicated in the development of various human cancers (4). MAPK mutations are common in ATC,

Received Jun. 27, 2018; revision accepted Nov. 15, 2018.

For correspondence or reprints contact: Roch-Philippe Charles, Institute for Biochemistry and Molecular Medicine, University of Bern, Bühlstrasse 28, $\mathrm{CH}-3012$ Bern, Switzerland.

E-mail: roch-philippe.charles@ibmm.unibe.ch

Published online Nov. 21, 2018.

COPYRIGHT (C 2019 by the Society of Nuclear Medicine and Molecular Imaging. with $25 \%$ of tumors harboring KRAS mutations, and depending on the study, $27 \%-45 \%$ are carrying BRAF mutations (5-7). The most common mutation found in BRAF codes for the protein kinase $\mathrm{BRAF}^{\mathrm{V} 600 \mathrm{E}}$, which induces constitutive activation of the downstream pathway (Fig. 1). Several important PI3'K pathway mutations in ATC include PTEN deletions (10\%-20\%) and PIK3CA mutations $(15 \%-25 \%)(8)$. We have previously shown that the $\mathrm{BRAF}^{\mathrm{V} 600 \mathrm{E}}$ mutation in combination with the PIK3CA ${ }^{\mathrm{H} 1047 \mathrm{R}}$ mutation induces tumor progression from papillary thyroid cancer (PTC) to ATC in a mouse model (9).

The link between alterations of the MAPK and PI3' K pathways and impairment of iodine metabolism is well documented, although the underlying mechanisms not fully elucidated. BRAF mutations are associated with impairment of NIS function and subsequent increased resistance to radioiodine uptake in $(10,11)$ thyroid cancers. Some studies suggest that BRAF inhibits the expression of PAX8 and prevents transcription of NIS (12).

The relationship between the MAPK pathway and the ability to store iodine intracellularly has been further demonstrated in mouse models of PTC, where selective MAPK pathway antagonists have been shown to increase the expression of NIS, with resultant increased uptake of iodine (13). Inhibition of the MAPK pathway via MEK is a promising strategy for treatment of iodine-refractory thyroid cancers and, specifically, ATC. Several studies suggest that $\mathrm{PI} 3^{\prime} \mathrm{K}$ inhibition increases radioiodine uptake in thyroid cancer cells $(14,15)$; however, this has not yet been evaluated in vivo. In this study, we aimed to investigate the consequences of pharmacologically targeting the MAPK and PI3'K pathways separately and in combination to induce radioiodine uptake in ATC cell lines and in a lethal double-mutant ATC $\left(\mathrm{BRAF}^{\mathrm{V} 600 \mathrm{E}}\right.$ PIK3CA $\left.{ }^{\mathrm{H} 1047 \mathrm{R}}\right)$ mouse model. We hypothesized that pharmacologic restoration of radioiodine uptake with subsequent radioactive iodine would be an effective means of therapy in a murine model of ATC.

\section{MATERIALS AND METHODS}

\section{Small-Molecule Kinases Inhibitors}

The inhibitors PD-325901 (MEK1/2), PLX-4032/vemurafenib $\left(\mathrm{BRAF}^{\mathrm{V} 600 \mathrm{E}}\right), \mathrm{PLX}^{-4720}\left(\mathrm{BRAF}^{\mathrm{V} 600 \mathrm{E}}\right.$ ), and GDC-0941 (class I PI3'K) were purchased from Abmole Bioscience. PLX-4032 was used in vitro as a BRAFV600E inhibitor, but because of poor solubility (16), PLX4720 was used for in vivo experiments.

\section{BRAF $^{\text {V600E }}$ PIK3CA ${ }^{\mathrm{H} 1047 \mathrm{R}}$ Mice}

Transgenic mice were bred by combining the following alleles as previously described (9): Braf CA (17), Pik3ca ${ }^{\text {Lat }}$ (18), and Thyro::Cre ${ }^{E R T 2}$ 


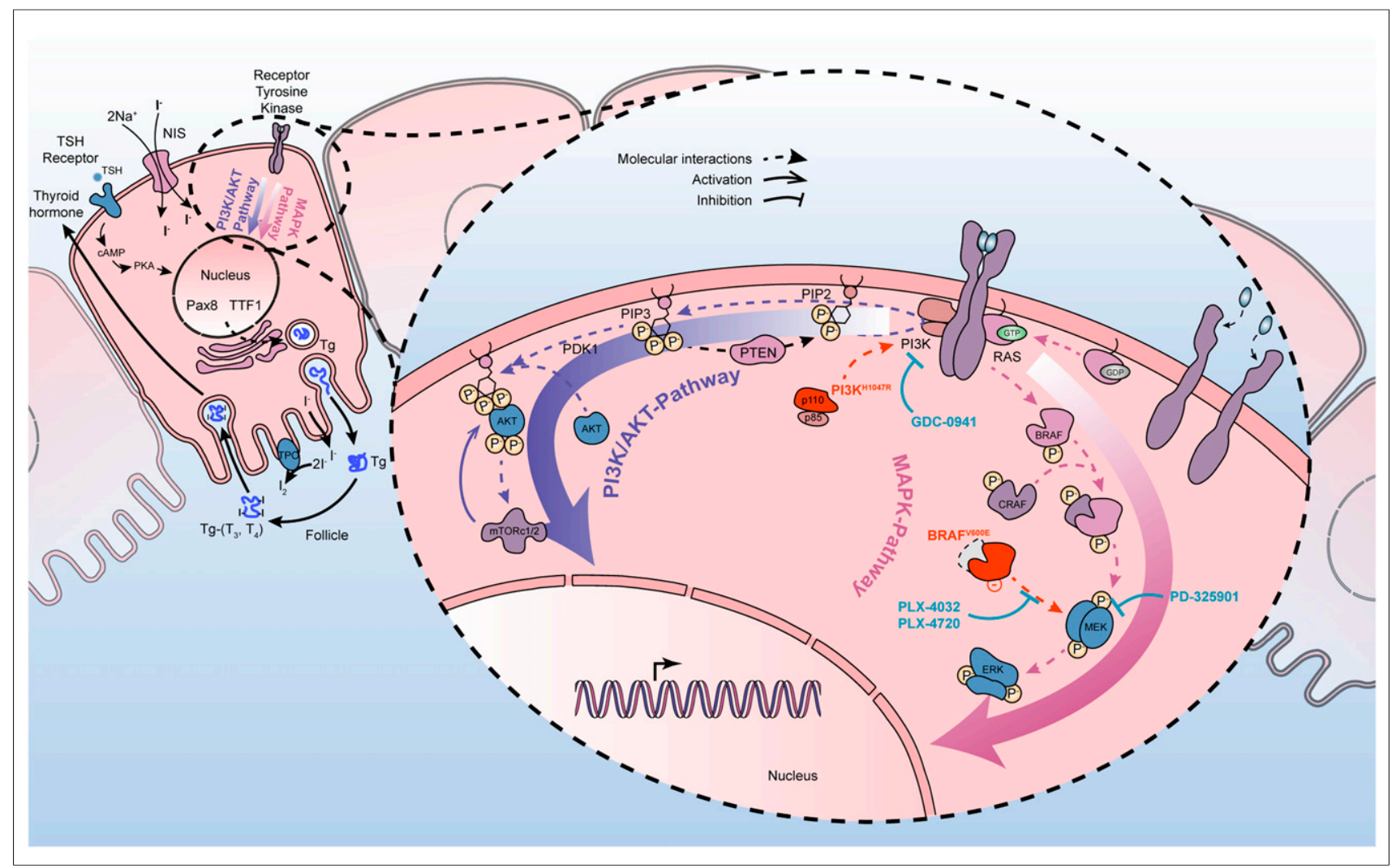

FIGURE 1. Mutationally activated pathways in the mouse model of thyroid cancer. (Left) Thyrocyte depicting key aspects of thyroid iodine transport and thyroid hormone synthesis. lodide $\left(I^{-}\right)$uptake is first and rate-limiting step in biosynthesis of thyroid hormone and is mediated by sodium iodide symporter (NIS). lodide is oxidized to iodine $\left(\mathrm{l}_{2}\right)$ by tyrosine peroxidase (TPO), which incorporates iodine into tyrosyl residues along thyroglobulin (Tg) backbone in colloid substance. Thyroglobulin fragments coupled to thyroid hormone enter thyrocyte by pinocytosis. Cleavage by endopeptidases results in release of T3 and T4 into blood. Thyroid-stimulating hormone (TSH) via its receptor stimulates these steps through activation of CAMP-PKA pathway and regulation of several transcription factors directly involved in thyroid hormone synthesis, such as PAX8 and TTF-1. (Right) MAPK and PI3'K pathways, including relevant mutations and drug targets in thyroid cancer. MAPK and PI3'K pathways are involved in cellular proliferation and differentiation, and activation of these pathways drives cell growth and decreases iodine metabolism. Mutation BRAFV600E (in red) stimulates MAPK pathway independent of upstream signal. Activation of RAS through binding of extracellular ligand to receptor tyrosine kinase leads to activation of PI3'K. From PIP3, phosphate is transferred to 3-phosphoinositide-dependent protein kinase 1 (PDK1), which in turn phosphorylates AKT, leading to its activation and translocation into nuclei, where it regulates transcription of target genes involved in growth. P-AKT in cytoplasm activates other targets such as mammalian target of rapamycin (mTOR), which is important in promoting protein translation and consequently cell growth. PTEN is phosphatase converting PIP3 to PIP2 and playing role of tumor suppressor as major negative regulatory mechanism of PI3'K/AKT pathway. Mutation PI3KCA H1047R results in hyperactivation of pathway. Pharmacologic inhibition of BRAFV600E occurs with PLX-4032/vemurafenib or PLX-4720, a preclinical analog. MEK is specifically inhibited by PD-325901, which leads to inhibition of ERK activity. $\mathrm{PI} 3^{\prime} \mathrm{K}$ is specifically inhibited by GDC-0941. PAX8 = paired box gene 8; TTF-1 = thyroid transcription factor 1 .

(19). Mutations were induced by 5 consecutive daily injections of $1 \mathrm{mg}$ of tamoxifen intraperitoneally. Mice were cared for in accordance with Swiss federal guidelines, housed in isolated ventilated cages, and fed ad libitum in a $12 \mathrm{~h} / 12 \mathrm{~h}$ cycle of light and dark. The experimental protocol was approved by the Bernese Cantonal Ethical Commission for Animal Experimentation (license number BE120/13).

\section{Cell Lines}

The human cell lines 8505c, Sw1736, and OCUT-2 were obtained and maintained as described previously (20).

\section{In Vitro Evaluation of ATC NIS Messenger RNA (mRNA) Expression}

Cells were seeded in 10-cm Petri dishes at $25 \%$ confluency overnight and then treated with PD-325901 at $10 \mathrm{nM}$, GDC-0941 at $100 \mathrm{nM}, \mathrm{PLX}-4032$ at $100 \mathrm{nM}$, and PD-325901 at $10 \mathrm{nM}$; with GDC0941 at $100 \mathrm{nM}$ or PLX-4032 at $100 \mathrm{nM}$; and with GDC-0941 at 100 $\mathrm{nM}$ at $37^{\circ} \mathrm{C}$ for $48 \mathrm{~h}$. Cells were washed twice with $5 \mathrm{~mL}$ of cold phosphate-buffered saline and then collected in $1 \mathrm{~mL}$ by scraping the dish surface. A centrifugation for $10 \mathrm{~min}$ at $3,000 \mathrm{~g}$ was performed, and the cell pellets were frozen in liquid nitrogen then stored at $-80^{\circ} \mathrm{C}$. NIS mRNA levels were evaluated by quantitative polymerase chain reaction.

\section{In Vivo Evaluation of Thyroid-Specific Gene Expression After Kinase Inhibition}

Thyroids from 8 single-mutant BRAF $^{\mathrm{V} 600 \mathrm{E}}$ mice $\left(\mathrm{Braf}^{\mathrm{CA} /+}\right.$; Thyro::Cre $\left.e^{E R T 2}\right)$ that had been treated by oral gavage for $21 \mathrm{~d}$ with a $12.5 \mathrm{mg} / \mathrm{kg}$ dose of PD-325901 $(n=4)$ or with $0.5 \%$ hydroxypropyl methylcellulose and $0.2 \%$ polysorbate- $80(n=4)$ were used as a control. These thyroid tissues were fixed in formalin and embedded in paraffin blocks (21), from which RNA was extracted with High Pure FFPET RNA isolation kit (Roche) according to the manufacturer's protocol.

Twenty-three BRAF ${ }^{\mathrm{V} 600 \mathrm{E}}$ PIK3CA ${ }^{\mathrm{H} 1047 \mathrm{R}}$ double-mutant mice $\left(\mathrm{Braf} \mathrm{CA}^{\mathrm{C}+}\right.$; Pik3 $\mathrm{Ca}^{\mathrm{Lat} /+}$; Thyro::Cre ${ }^{E R T 2}$ ) were treated 2 mo after tumor induction with PD-325901 at $5 \mathrm{mg} / \mathrm{kg}(n=4), \operatorname{PLX}-4720$ at $30 \mathrm{mg} / \mathrm{kg}(n=5)$, or 
GDC-0941 at $50 \mathrm{mg} / \mathrm{kg}(n=5)$ as single treatments, or with PLX/ GDC $(n=5)$ and PD/GDC $(n=4)$ in combination, by oral gavage daily for $10 \mathrm{~d}$. Additionally, thyroid glands from 12 Cre-negative mice $\left(\mathrm{Braf}^{\mathrm{CA} /+} ; \mathrm{Pik} \mathrm{Ca}^{\mathrm{Lat} /+}\right.$ ) were combined for a total of $n=3$ and used as nontumoral controls. Hydroxypropyl methylcellulose at $0.5 \%$ and polysorbate- 80 at $0.2 \%$ were used as a control. Mice were then sacrificed, thyroid glands were dissected and snap-frozen using liquid nitrogen, and total RNA was purified with the QIAzol reagent from Qiagen according to the manufacturer's instructions with a TissueLyser LT using iron beads. Nis, Tshr, Tg, Pax8, Tpo, and Ttf1 mRNA levels were evaluated.

\section{Quantitative Polymerase Chain Reaction}

A 500-ng quantity of RNA underwent reverse transcription using Oligo(dT)12-18 Primer and the Super Script II Reverse Transcriptase from Invitrogen following the manufacturer's suggested protocol. Samples were run on ViiA 7 real-time polymerase chain reaction system (Applied Biosystems, Life Technologies) using the TaqMan gene expression Master Mix and primers from Applied Biosystems. GAPDH was used for normalization using the $2^{\Delta \Delta \mathrm{Ct}}$ calculation method. All values were expressed as percentage of the baseline transcription levels found in nontumoral Cre-negative thyroid tissues. The following primers were purchased from Applied Biosystems, Life Technologies: NIS (Hs00166567_m1), Nis (Mm01351811_m1), Tg (Mm01200340_m1), Ttf1 (Mm00657018_m1), Pax8 (Mm00440623_m1), Tpo (Mm00456355_m1), Tshr (Mm00442027_m1), GAPDH (Hs02758991_g1), and Gapdh (Mm99999915_g1).

\section{In Vitro ${ }^{123}$ I Uptake Assay}

Cells were seeded in Corning 96-well plates at 30\% confluency, incubated overnight at $37^{\circ} \mathrm{C}$ and $5 \% \mathrm{CO}_{2}$, and treated for $48 \mathrm{~h}$ with various concentrations of PD-325901 $(0,0.1,1,10,100$, and 1,000 $\mathrm{nM}$ ) with or without $200 \mathrm{nM}$ GDC-0941. All except one of the PD-325901 concentrations used were below the Cmax value (200 nM), which corresponds to the maximal concentration in human plasma without toxicity recorded (22). Cells were washed with phosphate-buffered saline, and cell viability was assessed with the AlamarBlue Cell Viability Reagent (no. 765506, Invitrogen) by measuring fluorescence at $634 \mathrm{~nm}$ (SpectraMax M4 plate reader; Molecular Devices). After removal of AlamarBlue, cells were washed with phosphate-buffered saline and incubated with culture medium containing $18.5 \mathrm{kBq}$ of ${ }^{123} \mathrm{I}$ (GE Healthcare) for $1 \mathrm{~h}$ at $37^{\circ} \mathrm{C}$. The medium was removed, and the cells were washed 3 times with phosphate-buffered saline, which was then replaced by $50 \mu \mathrm{L}$ of scintillation reagent (MicroScint-20; PerkinElmer) per well. Activity was measured with a microplate scintillation counter (Packard Topcount NXT).

\section{In Vivo ${ }^{125}$ I Uptake Assay}

Eleven BRAF ${ }^{\mathrm{V} 600 \mathrm{E}}$ PIK3CA ${ }^{\mathrm{H} 1047 \mathrm{R}}$ double-mutant mice were treated daily by oral gavage for 2 wk with control $(n=4)$ or with PD325901 at $5 \mathrm{mg} / \mathrm{kg}$ alone $(n=4)$ or in combination with GDC-0941 at $30 \mathrm{mg} / \mathrm{kg}(n=3)$. Hydroxypropyl methylcellulose with $0.5 \%$ polysorbate- 80 was used as a control. Then, mice were injected via tail vein with $1.11 \mathrm{MBq}$ of ${ }^{125}$ I (ANAWA; Biomedical Services and Products) and euthanized $1 \mathrm{~h}$ later with $\mathrm{CO}_{2}$. Thyroid glands, salivary glands, liver, and blood were harvested and weighed. Radioactivity was quantified for $30 \mathrm{~s}$ per sample using a PerkinElmer 2470 automatic $\gamma$-counter.

\section{In Vivo Tumor Treatment Experiment}

16 BRAF $^{\mathrm{V} 600 \mathrm{E}}$ PIK3CA ${ }^{\mathrm{H} 1047 \mathrm{R}}$ double-mutant mice were treated daily by oral gavage for $10 \mathrm{~d}$ with control $(n=8)$ or PD-325901 at $5 \mathrm{mg} / \mathrm{kg}(n=8)$. Afterward, half the mice in each group were injected with $18.5 \mathrm{MBq}$ of ${ }^{131} \mathrm{I}$ (PerkinElmer) via tail vein. Tumor volume was assessed weekly for $9 \mathrm{wk}$ with the following previously described protocol (20). Tumor burden was determined by measuring the surface of the largest thyroid cross section normalized to the size of the gland at the beginning of the study. In this model of thyroid cancer, all thyrocytes are undergoing molecular changes leading to cancerization of the entire thyroid gland. Therefore, the assessment of whole thyroid size for tumor burden is a reliable and wellaccepted approximation $(9,21,23)$.

\section{Statistical Analysis}

A 2-way ANOVA test was performed to evaluate the difference in tumor burden measurement between treatment groups. A 1-way ANOVA test was used to evaluate the difference in NIS mRNA levels in vitro, the difference in radioiodine uptake in vivo, and the difference between treatments on mRNA levels of different genes in vivo (followed by the Tukey multiple-comparisons test). All statistical analyses were performed using GraphPad Prism. $P$ values equal to or less than 0.05 were considered significant.

\section{RESULTS}

\section{MEK Inhibition Alone or in} Combination with PI3'K Inhibition Enhances NIS mRNA Transcription and Increases Intracellular lodine Transport In Vitro

The effect of the MEK inhibitor PD325901 and the BRAF inhibitor PLX-4032

FIGURE 2. MEK inhibition led to elevation of NIS mRNA levels and iodine uptake in vitro. (A) NIS mRNA levels in 2 ATC cell lines, $8505 \mathrm{c}$ and OCUT-2, treated for $48 \mathrm{~h}$ with PD at $100 \mathrm{nM}$ alone, $\mathrm{PLX}$ at $1 \mu \mathrm{M}$ alone, or in combination with GDC at $1 \mu \mathrm{M}$. Values are normalized to untreated cells. (B) ${ }^{123}$ uptake in 3 ATC cell lines treated with different concentrations of PD alone or in combination with GDC. Values are normalized to untreated cells. $\mathrm{C}_{\max }$ indicates maximum plasma concentration of PD in patients without side effects. 
(vemurafenib) alone or combined with the PI3'K inhibitor GDC0941 was evaluated on mRNA transcription levels of NIS in $8505 \mathrm{c}$ and OCUT-2, 2 ATC cell lines harboring a BRAF ${ }^{\mathrm{V} 600 \mathrm{E}}$ mutation (Fig. 2A). In 8505c, PD-325901 treatment alone induced a 4-fold increase in NIS mRNA level after $48 \mathrm{~h}$, and the addition of GDC-0941 did not further augment NIS mRNA expression. GDC-0941 alone did not have an effect on NIS transcription, and PLX-4032 did not lead to a significant elevation of NIS mRNA expression when it was applied alone or in combination with GDC-0941. The OCUT-2 cell line, which has a PIK3CA ${ }^{\mathrm{H} 1047 \mathrm{R}}$ mutation in addition to a BRAFV600E mutation, showed similar upregulation of NIS mRNA transcription after treatment with PD-325901, with an additional response to GDC-0941 alone (3-fold increase in NIS mRNA level) and with the combination of PD325901 and GDC-0941 (9-fold increase). In OCUT-2, PLX-4032 alone or in combination with GDC-0941 resulted in a 5-fold increase in NIS mRNA levels.

After $48 \mathrm{~h}$ of treatment with PD-325901 at varying concentrations ranging from 0 to $1 \mu \mathrm{M}$, cellular iodine uptake increased by more than double in OCUT-2 and 8505c, as well as in SW1736, a third ATC cell line also harboring a BRAF ${ }^{\mathrm{V} 600 \mathrm{E}}$ mutation.

Addition of 200 nM GDC-0941 with PD-325901 further increased uptake of ${ }^{123} \mathrm{I}$ in the OCUT-2 and SW1736 cell lines, but not in 8505c (Fig. 2B).

\section{MEK Inhibition but Not BRAF ${ }^{\mathrm{V} 600 E}$ Inhibition Increases Nis, Tshr, Tg, Pax8, and Tpo mRNA Transcription In Vivo, Which Correlates with Histologic Renormalization of Thyroid Follicles}

A 4-fold increase in Nis mRNA levels extracted from formalinfixed, paraffin-embedded tumor sections in $\mathrm{BRAF}^{\mathrm{V} 600 \mathrm{E}}$ single-mutant mice (21) treated with the MEK inhibitor PD-325091, as compared with control mice (Fig. 3A), was observed. Additionally, the treated group showed restoration of normal thyroid follicular structure (near-spheroid follicles containing colloid substance) on hematoxylin-eosin staining (Fig. 3B), whereas the control group displayed a papillary structure and absence of normal thyroid follicles.

We then switched to a more aggressive murine $\mathrm{BRAF}^{\mathrm{V} 600 \mathrm{E}}$ PIK3CA ${ }^{\text {H1047R }}$ double-mutant model. In animals treated with the MEK inhibitor PD-325901, there was upregulation of Nis mRNA by almost 20 -fold. The selective BRAF ${ }^{\mathrm{V} 600 \mathrm{E}}$ inhibitor PLX-4720 did not increase Nis mRNA transcription, nor did the PI3'K inhibitor GDC-0941
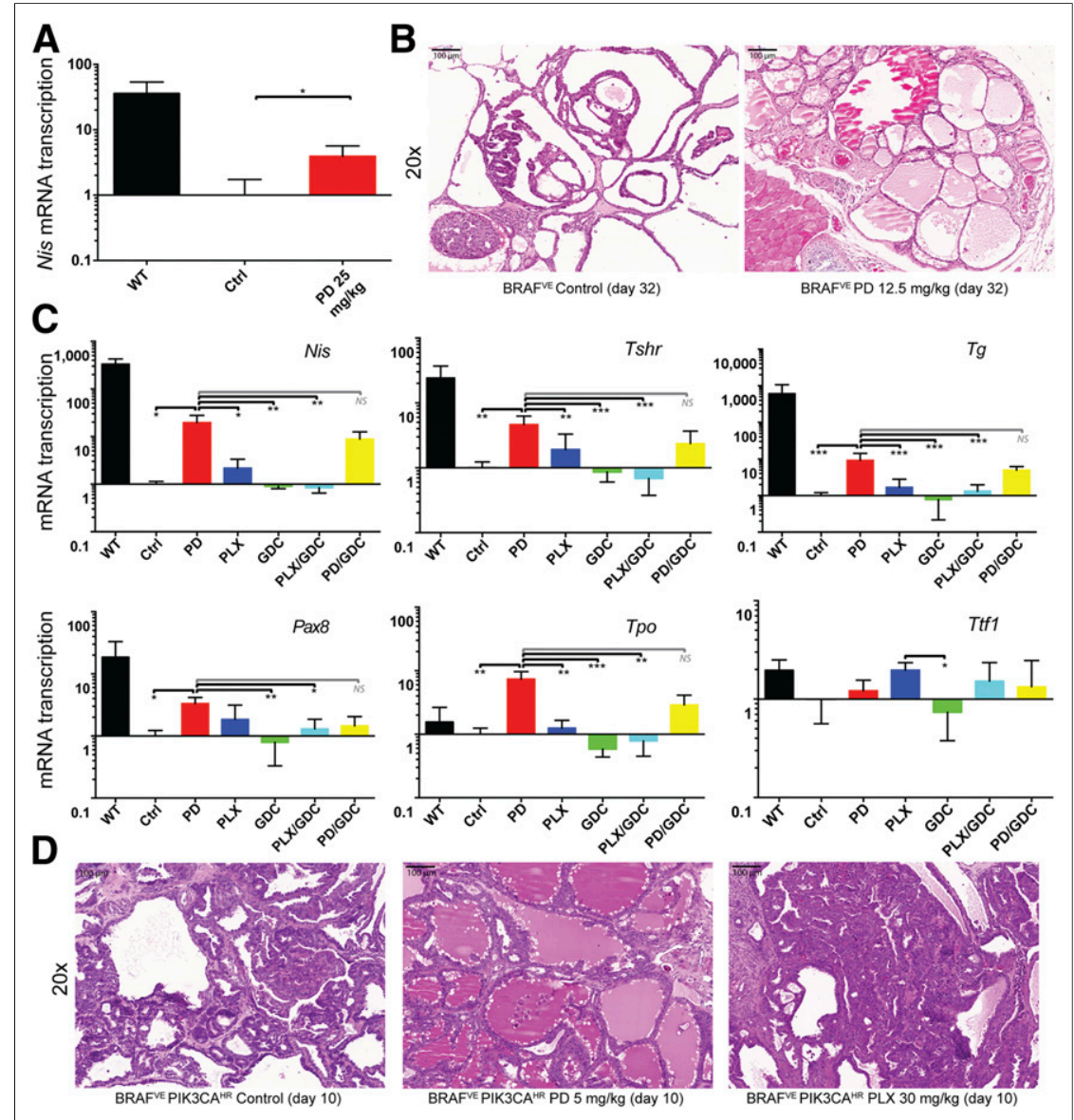

FIGURE 3. MEK inhibition but not BRAF ${ }^{\mathrm{V} 600 E}$ inhibition induced restoration of Nis, $T s h r$, and $T g$ transcripts and resulted in near-normalization of follicular organization in vivo. (A) Nis mRNA levels in BRAF ${ }^{\mathrm{V} 600 \mathrm{E}}$ single-mutant mice treated with $\mathrm{PD}$ or control. (B) Hematoxylin- and eosinstained thyroid sections. (C) mRNA expression levels of Nis, Tshr, Tg, Pax8, Tpo, and Ttf1 normalized to normal levels in double-mutant mice (BARFV600E PIK3CA ${ }^{\mathrm{H} 1047 \mathrm{R}}$ ) treated with control, PD, PLX, GDC, PLX/GDC, or PD/GDC for 10 d. (D) Hematoxylin- and eosin-stained thyroid sections from mice after treatments. alone or in combination with PD-325901 or PLX-4720 (Fig. 3C).

Evaluation of mRNA transcription of additional genes known to be important in thyroid gland function was performed, including $T$ shr, Pax8, Tg, Tpo, and Ttfl. For all genes except $T t f-1$, the same pattern as Nis was observed, with a strong response to PD-325901, no further elevation in combination with GDC-0941, and little response to any of the other drugs alone (Fig. 3C). Elevation of protein expression could be confirmed in immunohistochemistry staining for TG, PAX8, and TTF-1 (Supplemental Fig. 1; supplemental materials are available at http://jnm.snmjournals.org) (24).

Increased Nis, Tshr, Tg, Pax8, and Tpo transcription correlated with histologic nearnormalization in the appearance of thyroid follicles after $10 \mathrm{~d}$ of PD-325901 treatment on hematoxylin-eosin, a finding that was not replicated in control mice or mice treated with PLX-4720 and GDC-0941 (Fig. 3D).

\section{MEK Inhibition Leads to a Significant Increase in Thyroid Radioiodine Uptake in BRAF ${ }^{\mathrm{V} 600 E}$ PIK3CA $^{\mathrm{H} 1047 \mathrm{R}}$ Double-Mutant Mice}

After gene expression analyses, a validation of functional changes in radioiodine uptake in BRAFV600E PIK3CA ${ }^{\text {H1047R }}$ doublemutant mice using PD-325901 alone and in combination with GDC-0941 was attempted. After $14 \mathrm{~d}$ of daily treatment with PD325901 with or without GDC-0941, there was a 2.5 -fold increase in ${ }^{125}$ I thyroid uptake in $\mathrm{BRAF}^{\mathrm{V} 600 \mathrm{E}} \mathrm{PIK} 3 \mathrm{CA}^{\mathrm{H} 1047 \mathrm{R}}$ double-mutant mice compared with controls. There was 


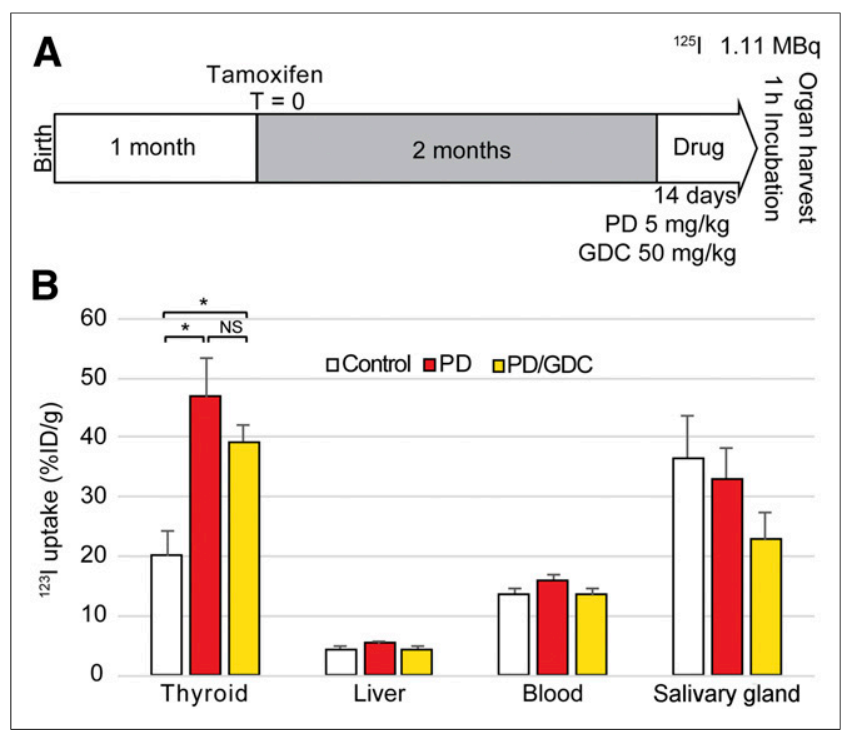

FIGURE 4. MEK inhibition led to increased radioactive iodine uptake by thyroid in BRAF ${ }^{\mathrm{V} 600 \mathrm{E}}$ PIK3CA $\mathrm{H}^{\mathrm{H} 1047 \mathrm{R}}$ double-mutant mice. (A) Schematic representation of in vivo radioiodine uptake experiment. (B) Uptake expressed as percentage injected dose of ${ }^{125}$ / per gram of tissue in thyroid, salivary glands, liver, and blood harvested from mice treated with control, PD alone, or PD in combination with GDC for 2 wk.

no significant difference in thyroid radioiodine uptake between mice treated with PD-325901 or those treated with a combination of PD-325901 and GDC-0941. Liver uptake, used to assess background uptake because of passive diffusion through the apical membrane, remained stable across the different treatment regimens. The level of ${ }^{125} \mathrm{I}$ in the blood was approximately $15 \%$ injected dose per gram and did not significantly change with the different treatments. The salivary glands, which express NIS but are not able to store iodine because of lack of TG expression, showed slightly decreased uptake with combination treatment (Fig. 4).

\section{MEK Inhibitor Treatment Before ${ }^{131}$ | Therapy Reduces Tumor Burden in BRAF ${ }^{\mathrm{V} 600 \mathrm{E}} \mathrm{PIK} \mathrm{CA}^{\mathrm{H} 1047 \mathrm{R}}$ Double-Mutant Mice}

An evaluation of the translation from the observed increase in radioiodine uptake in $\mathrm{BRAF}^{\mathrm{V} 600 \mathrm{E}} \mathrm{PIK} 3 \mathrm{CA}^{\mathrm{H} 1047 \mathrm{R}}$ double-mutant mice treated with MEK inhibition via PD-325901 into reduced tumor burden when injected with therapeutic radioiodine $\left({ }^{131} \mathrm{I}\right)$ was performed. The treatment study design, including tumor induction, treatment with PD-325901, and radioiodine therapy, is depicted in Figure 5A. The control group displayed progressive tumor growth of approximately $15 \%$ each week. Mice receiving a 10-d course of treatment with PD-325901 experienced only an initial reduction in tumor burden, with the therapeutic effect disappearing immediately after treatment cessation. The group of mice injected with radioiodine alone demonstrated a stable tumor burden for $6 \mathrm{wk}$ before tumors eventually increased in size. Mice treated with a 10-d course of PD-325901 followed by injection with ${ }^{131}$ I displayed a reduction in the tumor burden by $60 \%$ of the initial size $6 \mathrm{wk}$ after iodine injection and remained stable for 3 wk (Fig. 5B).

\section{DISCUSSION}

Important genes in thyroid hormone synthesis such as NIS, $T S H R, T G$, and TPO are frequently downregulated in advanced thyroid cancer. The loss of this machinery is progressive, beginning with NIS and then involving TPO, TG, and TSHR (25-27).

In the present study, we showed in vitro evidence that MEK inhibition induced robust NIS mRNA transcription in human ATC cell lines harboring a BRAF ${ }^{\mathrm{V} 600 \mathrm{E}}$ mutation, which resulted in elevated cellular ${ }^{123}$ I uptake. We also showed that this result could be translated in vivo in a mouse model of lethally aggressive thyroid cancer by significantly elevating the radioiodine uptake, resulting in stable reduction of tumor burden.

We have previously shown that MEK inhibition in combination with $\mathrm{PI}^{\prime} \mathrm{K}$ inhibition could lead to a profound reduction driven by apoptosis (28). This treatment, although promising, has the disadvantage of synergistic side effects associated with both drugs. Moreover, after treatment cessation, a rapid regrowth of tumors occurred. In the present study, a short period of drug treatment (10 d) enabled a potentially curative treatment with radioiodine.

In vivo, MEK inhibition in a BRAFV600E single-mutant mouse model resulted in a Nis 4-fold transcription elevation (Fig. 3A).
FIGURE 5. Combination of MEK inhibition with ${ }^{131}$ l led to profound reduction in tumo burden in BRAFV600E PIK3CA ${ }^{\mathrm{H} 1047 \mathrm{R}}$ double-mutant mice. (A) Schematic representation of in vivo treatment study. (B) Thyroid tumor burden measured weekly by ultrasound expressed as percentage of pretreatment tumor burden in mice. (C) Representative gray-scale ultrasound images of thyroid gland at $77 \mathrm{~d}$ after treatment from mice in 4 different treatment cohorts. 
This iodine induction in a PTC model is consistent with the current literature $(13,29,30)$. In a mouse model of more advanced thyroid cancer harboring $\mathrm{BRAF}^{\mathrm{V} 600 \mathrm{E}}$ and PIK3CA $\mathrm{A}^{\mathrm{H} 1047 \mathrm{R}}$ mutations (Fig. 3C), we demonstrated that a MEK inhibition induced a strong elevation of mRNA levels of genes important in thyroid hormone synthesis, such as Nis (6-fold), Tshr, Tg, Pax8, and Tpo. Interestingly, the combination of MEK inhibition with PI3' $\mathrm{K}$ inhibition did not provide further upregulation of the tested genes. Specific BRAF ${ }^{V 600 E}$ inhibition did not induce an increase in any of these transcripts (Fig. 3C). This is concordant with Nagarajah et al., who showed that the more profound the MEK inhibition is, the stronger is the NIS reexpression (30). It was previously demonstrated that BRAF-specific inhibition can induce only transient ERK dephosphorylation and that the signal is restored with time through the wild-type allele of BRAF (31). Additionally, our

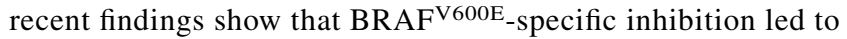
a paradoxic activation of ERK in the double-mutant mouse model (20). Furthermore, there are very few data reporting vemurafenib treatment in ATC, and one study showed mitigated results, including one case of progression under $\mathrm{BRAF}^{\mathrm{V} 600 \mathrm{E}}$ inhibitor (32).

On a functional level, a nearly 2.5 -fold increase in radioiodine uptake in the thyroids of mice treated with a MEK inhibitor as compared with mice receiving the control treatment was observed (Fig. 4) after only $10 \mathrm{~d}$ of PD-325901 treatment. Increased radioiodine uptake translated into a stable, profound reduction in tumor burden (Fig. 5). Mice treated with radioiodine showed only an initial stabilization of the tumor burden followed by a rapid tumor growth. This short-lasting response to radioiodine could be explained by tumor heterogeneity, with regions of untransformed cells and PTC. As there was no added benefit to using a drug combination with a PI3' $\mathrm{K}$ inhibitor even in tumors harboring a PIK3CA ${ }^{\text {H1047R }}$ mutation (Fig. 4), this was not tested in the context of curative radioiodine.

\section{CONCLUSION}

Our study shows that MEK inhibition as a single-drug treatment is the most efficient regimen to induce the expression of protein machinery required for iodine uptake in human ATC cell lines and in our aggressive murine model of thyroid cancer. Until now, most studies have focused mainly on PTC mouse models driven by a single BRAFV600E mutation (13). Here, we provide new evidence that the RAS-MEK-ERK axis is essential for NIS expression even in ATC cell lines and in a clinically relevant mouse model carrying the 2 mutations $\mathrm{BRAF}^{\mathrm{V} 600 \mathrm{E}}$ and PIK3CA ${ }^{\mathrm{H} 1047 \mathrm{R}}$. We performed a metaanalysis of the 3 available studies conducting genetic profiling in ATC patients and found that the occurrence of both BRAF and PIK3CA mutations is found in $12 \%$ of ATC patients (Supplemental Table 1 (5-7)). Thus, we believe that the present results represent an encouraging initial step toward the successful treatment of non-radioiodine-responsive thyroid cancers.

\section{DISCLOSURE}

Funding support was provided by Swiss National Science Foundation grant 31003A_149824/1 and Swiss National Science Foundation grant NCCR-TransCure. No other potential conflict of interest relevant to this article was reported.

\section{ACKNOWLEDGMENTS}

We thank Prof. Martin McMahon for his mentorship and for providing the BRAF mutant mice. We thank Prof. Engelhard, Dr. Deutsch, and Dr. Benarafa for permission to use the vivarium at the Theodor Kocher Institute. We thank Dr. Didier Colin for his help with Figure 1. Also, we acknowledge the Microscopy Imaging Center of the University of Bern (MIC) for their contributions to this study. Oussama ElMokh and Matthias Roelli were enrolled in the Graduate School for Cellular and Biomedical Research (GCB) of the University of Bern during this study.

\section{REFERENCES}

1. Kebebew E, Greenspan FS, Clark OH, Woeber KA, McMillan A. Anaplastic thyroid carcinoma: treatment outcome and prognostic factors. Cancer. 2005;103: 1330-1335.

2. Gilliland FD, Hunt WC, Morris DM, Key CR. Prognostic factors for thyroid carcinoma: a population-based study of 15,698 cases from the Surveillance, Epidemiology and End Results (SEER) program 1973-1991. Cancer. 1997;79: 564-573.

3. Mazzaferri EL. An overview of the management of papillary and follicular thyroid carcinoma. Thyroid. 1999;9:421-427.

4. Kim EK, Choi E. Pathological roles of MAPK signaling pathways in human diseases.Biochim Biophys Acta.2010;1802:396-405.

5. Kunstman JW, Christofer Juhlin C, Goh G, et al. Characterization of the mutational landscape of anaplastic thyroid cancer via whole-exome sequencing. Hum Mol Genet. 2015;24:2318-2329.

6. Pozdeyev N, Gay LM, Sokol ES, et al. Genetic analysis of 779 advanced differentiated and anaplastic thyroid cancers. Clin Cancer Res. 2018;24:3059-3068.

7. Landa I, Ibrahimpasic T, Boucai L, et al. Genomic and transcriptomic hallmarks of poorly differentiated and anaplastic thyroid cancers. J Clin Invest. 2016;126: 1052-1066.

8. Xing M. Molecular pathogenesis and mechanisms of thyroid cancer. Nat Rev Cancer. 2013;13:184-199.

9. Charles R-P, Silva J, Iezza G, Phillips WA, McMahon M. Activating BRAF and PIK3CA mutations cooperate to promote anaplastic thyroid carcinogenesis. Mol Cancer Res. 2014;12:979-986.

10. Riesco-Eizaguirre G, Gutiérrez-Martínez P, García-Cabezas M, Nistal M, Santisteban P. The oncogene BRAFV600E is associated with a high risk of recurrence and less differentiated papillary thyroid carcinoma due to the impairment of $\mathrm{Na}+/ \mathrm{I}-$ targeting to the membrane. Endocr Relat Cancer. 2006;13:257269.

11. Romei C, Ciampi R, Faviana P, et al. BRAFV600E mutation, but not RET/PTC rearrangements, is correlated with a lower expression of both thyroperoxidase and sodium iodide symporter genes in papillary thyroid cancer. Endocr Relat Cancer. 2008;15:511-520.

12. Costamagna E, García B, Santisteban P. The functional interaction between the paired domain transcription factor Pax8 and Smad3 is involved in transforming growth factor- $\beta$ repression of the sodium/iodide symporter gene. $J$ Biol Chem. 2004;279:3439-3446.

13. Chakravarty D, Santos E, Ryder M, et al. Small-molecule MAPK inhibitors restore radioiodine incorporation in mouse thyroid cancers with conditional BRAF activation. J Clin Invest. 2011;121:4700-4711.

14. Liu Y-Y, Zhang X, Ringel MD, Jhiang SM. Modulation of sodium iodide symporter expression and function by LY294002, Akti-1/2 and rapamycin in thyroid cells. Endocr Relat Cancer. 2012;19:291-304.

15. Bozorg-Ghalati F, Hedayati M, Dianatpour M, Azizi F, Mosaffa N, Mehrabani D. Effects of a phosphoinositide-3-kinase inhibitor on anaplastic thyroid cancer stem cells. Asian Pac J Cancer Prev. 2017;18:2287-2291.

16. Shah N, Iyer RM, Mair H-JJ, et al. Improved human bioavailability of vemurafenib, a practically insoluble drug, using an amorphous polymer-stabilized solid dispersion prepared by a solvent-controlled coprecipitation process. J Pharm Sci. 2013;102:967-981.

17. Dankort D, Filenova E, Collado M, Serrano M, Jones K, McMahon M. A new mouse model to explore the initiation, progression, and therapy of BRAFV600Einduced lung tumors. Genes Dev. 2007;21:379-384.

18. Kinross KM, Montgomery KG, Kleinschmidt M, et al. An activating Pik3ca mutation coupled with Pten loss is sufficient to initiate ovarian tumorigenesis in mice. J Clin Invest. 2012;122:553-557. 
19. Undeutsch H, Löf C, Offermanns S, Kero J. A mouse model with tamoxifeninducible thyrocyte-specific cre recombinase activity. Genesis. 2014;52:333-340.

20. Roelli MA, Ruffieux-Daidié D, Stooss A, et al. PIK3CAH1047R-induced paradoxical ERK activation results in resistance to BRAFV600E specific inhibitors in BRAFV600E PIK3CAH1047R double mutant thyroid tumors. Oncotarget. 2017;8:103207-103222.

21. Charles RP, Iezza G, Amendola E, Dankort D, McMahon M. Mutationally activated BRAFV600E elicits papillary thyroid cancer in the adult mouse. Cancer Res. 2011;71:3863-3871.

22. Haura EB, Ricart AD, Larson TG, et al. A phase II study of PD-0325901, an oral MEK inhibitor, in previously treated patients with advanced non-small cell lung cancer. Clin Cancer Res. 2010;16:2450-2457.

23. McFadden DG, Vernon A, Santiago PM, et al. p53 constrains progression to anaplastic thyroid carcinoma in a Braf-mutant mouse model of papillary thyroid cancer. Proc Natl Acad Sci USA. 2014;111:E1600-E1609.

24.. Berezowska S, Galván JA. Immunohistochemical detection of the autophagy markers LC3 and p62/SQSTM1 in formalin-fixed and paraffin-embedded tissue. Methods Mol Biol. 2017;1560:189-194.

25. Lazar V, Bidart JM, Caillou B, et al. Expression of the Na+/I- symporter gene in human thyroid tumors: a comparison study with other thyroid-specific genes. J Clin Endocrinol Metab. 1999;84:3228-3234.
26. Gérard AC, Daumerie C, Mestdagh C, et al. Correlation between the loss of thyroglobulin iodination and the expression of thyroid-specific proteins involved in iodine metabolism in thyroid carcinomas. J Clin Endocrinol Metab. 2003;88: 4977-4983.

27. Russo D, Damante G, Puxeddu E, Durante C, Filetti S. Epigenetics of thyroid cancer and novel therapeutic targets. J Mol Endocrinol. 2011;46: R73-R81.

28. ElMokh O, Ruffieux-daidié D, Roelli MA, et al. Combined MEK and Pi3'-kinase inhibition reveals synergy in targeting thyroid cancer in vitro and in vivo. Oncotarget. 2017;8:24604-24620.

29. Ho AL, Grewal RK, Leboeuf R, et al. Selumetinib-enhanced radioiodine uptake in advanced thyroid cancer. $N$ Engl J Med. 2013;368:623-632.

30. Nagarajah J, Le M, Knauf JA, et al. Sustained ERK inhibition maximizes responses of BrafV600E thyroid cancers to radioiodine. J Clin Invest. 2016;126: 4119-4124.

31. Hatzivassiliou G, Song K, Yen I, et al. RAF inhibitors prime wild-type RAF to activate the MAPK pathway and enhance growth. Nature. 2010;464:431435.

32. Hyman DM, Puzanov I, Subbiah V, et al. Vemurafenib in multiple nonmelanoma cancers with BRAF V600 mutations. N Engl J Med. 2015;373:726736. 